\title{
Desenvolvimento de Operador Matemático para Algoritmos de Otimização Heurísticos Aplicado a Problema de Geoprospecção
}

\author{
W.P. CALIXTO ${ }^{1 *}$, T.M. PEREIRA ${ }^{2}$, J.C. MOTA ${ }^{3}$, A.J. ALVES ${ }^{1}$, \\ E.G. DOMINGUES ${ }^{1}$, J.L. DOMINGOS ${ }^{1}$, A.P. COIMBRA ${ }^{4}$ e B. ALVARENGA ${ }^{2}$
}

Recebido em 20 outubro, 2012 / Aceito em 5 junho, 2014

\begin{abstract}
RESUMO. O propósito deste trabalho é apresentar um operador genético desenvolvido a partir dos métodos matemáticos de extrapolação de curva. Este operador irá auxiliar na produção de um indivíduo melhor adaptado na população do algoritmo genético com codificação real, reconhecendo padrões inerentes aos genes dos cromossomos dos melhores indivíduos de cada geração. O operador proposto em conjunto com o algoritmo genético de codificação real é comparado com cinco outros métodos diferentes de otimização aplicados a prospecção geoelétrica.
\end{abstract}

Palavras-chave: otimização, operador matemático, algoritmo genético, codificação real.

\section{INTRODUÇÃO}

Vários são os métodos de otimização existentes na literatura. Estes métodos podem ser divididos em dois grupos distintos; i) métodos determinísticos e ii) métodos heurísticos. Os algoritmos de otimização determinísticos nem sempre conseguem resolver problemas com alta ordem de complexidade, gastando elevado tempo de execução para obter resultados satisfatórios, em alguns casos inviabilizando o processo de otimização [1].

Neste trabalho, um algoritmo genético (AG) é utilizado como método de otimização [2, 3]. Os AG são métodos estocásticos de busca cega de soluções otimizadas [4]. Eles não dispõem de nenhum conhecimento específico do problema a ser resolvido, de maneira que a busca é efetuada exclusivamente utilizando o valor da função de avaliação [5]. Eles não trabalham diretamente

\footnotetext{
*Autor correspondente: Wesley Pacheco Calixto.

${ }^{1}$ Núcleo de Estudos e Pesquisas Experimentais e Tecnológicas (NExT), Instituto Federal de Goiás (IFG), 74055-110 Goiânia, GO, Brasil.

E-mails:wpcalixto@ieee.org; aylton.alves@ifg.edu.br; elder.domingues@ifg.edu.br; jose.domingos@ifg.edu.br

${ }^{2}$ Escola de Engenharia Elétrica, Mecânica e de Computação (EMC), Universidade Federal de Goiás (UFG), 74605-010 Goiânia, GO, Brasil. E-mails: thiagothmp@uol.com.br; bernardo@eee.ufg.br

${ }^{3}$ Instituto de Matemática e Estatística (IME), Universidade Federal de Goiás (UFG), 74001-970 Goiânia, GO, Brasil.

E-mail: jesusdamota@gmail.com

${ }^{4}$ Institute of Systems and Robotics (ISR), University of Coimbra, Coimbra, 3030-290 Portugal.

E-mail: acoimbra@deec.uc.pt
} 
sobre o domínio do problema, mas sobre a representação de seus parâmetros, buscando simultaneamente entre o conjunto de possíveis soluções, um candidato otimizado, proporcionando controle sobre a busca em direção ao ótimo [6, 7].

O AG mantêm uma população de indivíduos que evoluem de acordo com as regras da teoria de seleção natural, manipulando operadores genéticos dos mais variados tipos. A aptidão de cada indivíduo é calculada pela função de avaliação $F(w)$, onde $w=\left[w_{1}, w_{2}, \ldots, w_{n}\right]$ é o vetor de parâmetros a serem otimizados. O AG em conjunto com um simulador do problema real explora as informações disponíveis de cada indivíduo. A Figura 1 ilustra o processo de otimização heurístico.

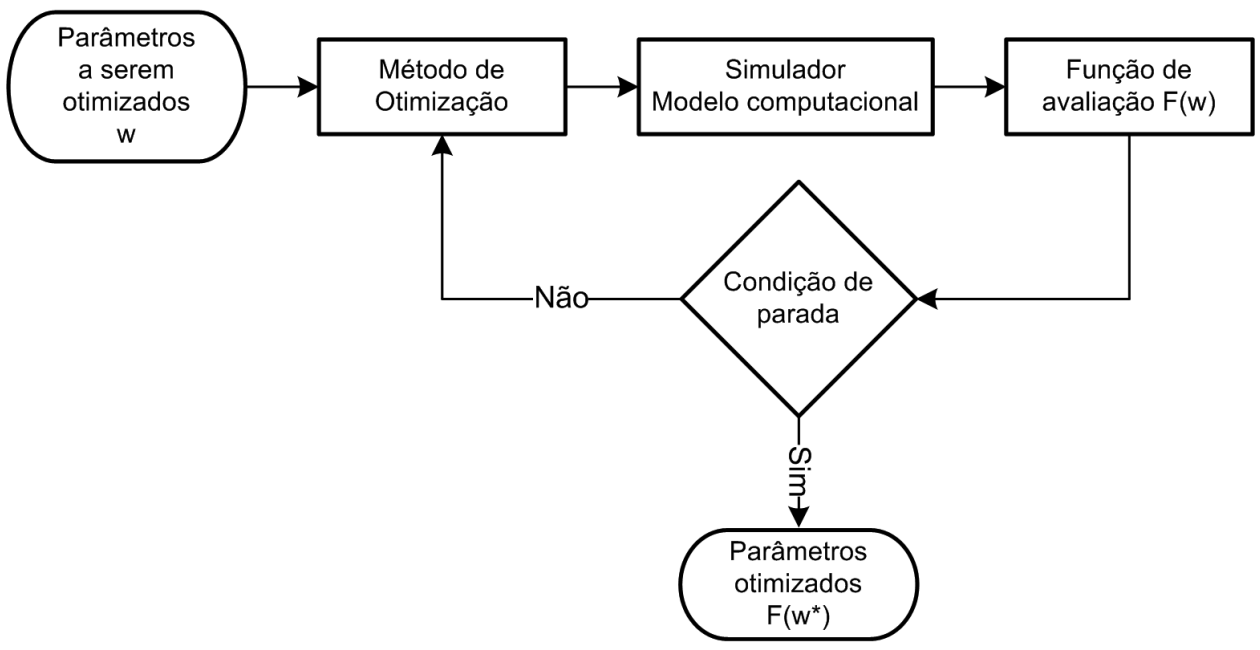

Figura 1: Processo de otimização.

Os operadores genéticos são necessários para que a população se diversifiquem e mantenham características de adaptação adquiridas pelas gerações anteriores. Os AG são robustos na solução de problemas de alta complexidade [8].

O intuito deste trabalho é apresentar um operador matemático que aplicado em conjunto com os demais operadores do AG, será capaz de acelerar o processo de otimização mantendo a diversidade ativa durante a busca em direção ao ponto otimizado $F\left(w^{*}\right)$. Na busca dos valores otimizados $F\left(w^{*}\right)$, será utilizado o algoritmo genético com codificação real clássico (AGCR) e as dificuldades encontradas serão contornadas utilizando o operador matemático proposto. Este operador é baseado na extrapolação dos genes de cada cromossomo pela função de avaliação $F(w)$. O operador proposto em conjunto com o AGCR será comparado com algumas metodologias difundidas de otimização aplicada ao processo de prospecção geoelétrico.

Na Seção 2 é apresentado o algoritmo genético com codificação real clássico. A Seção 3 descreve a ideia básica do operador proposto. Na Seção 4 faz-se uma descrição dos problemas de prospecção geoelétricos. Na Seção 5 são apresentados os resultados dos problemas e é realizada a comparação entre cinco metodologias de otimização utilizadas em problemas de prospecção geoelétrico. 


\section{Algoritmo Genético Clássico - Codificação Real}

Neste trabalho os problemas de otimização considerados são da forma:

$$
\begin{array}{ll} 
& \min F(w) \\
\text { sujeito a: } & w \in \Omega \subset \mathbf{R}^{p}
\end{array}
$$

onde $F$ é uma função definida em $R^{p}$ e $\Omega$ é o conjunto das possíveis soluções do problema. Uma solução $w^{*}$ de (2.1) é um ponto de mínimo global de $F$ em $\Omega$. Para resolver o problema da forma (2.1) utilizando o AG proposto, as variáveis $w \in \Omega$ são identificadas como cromossomos (indivíduos), e cada cromossomo é representado por um vetor de números binários, inteiros ou reais.

A técnica clássica de codificação do AG utiliza-se strings de bits a fim de representar os cromossomos. Porém, com a necessidade de maior precisão numérica, as cadeias de bits se tornam excessivamente longas, levando a esforço computacional maior em relação ao AGCR, e com isto o aumento do tempo de execução. Com a utilização do AGCR torna-se possível fazer maior exploração em tempo menor, o que é difícil de conseguir com as cadeias binárias [9]. Duas outras vantagens na utilização da codificação real são: i) a capacidade de explorar gradualmente as funções com variáveis contínuas e ii) nas cadeias binárias existe a necessidade da conversão dos bits para valor numérico.

Neste trabalho o AGCR utiliza o Simple Crossover e o Non-Uniform Mutation [3], conforme descrito a seguir.

Simple Crossover: Sejam $l_{i}$ e $l_{j}$ dois cromossomos da população corrente, $l_{i}=\left[\begin{array}{lll}c_{i 1} & c_{i 2} & \ldots\end{array}\right.$ $\left.c_{i(m-1)} c_{i m}\right]$ e $l_{j}=\left[\begin{array}{lllll}c_{j 1} & c_{j 2} & \ldots & c_{j(m-1)} & c_{j m}\end{array}\right]$ escolhidos aleatoriamente. Escolhe-se uma variável probabilística binária de decisão $\tau$, de tal modo que, se $\tau=1$ trocam-se os genes $c_{i m}$ por $c_{j m}$, obtendo-se dois novos cromossomos $l_{i}^{\prime}=\left[\begin{array}{lllll}c_{i 1} & c_{i 2} & \ldots & c_{i(m-1)} & c_{j m}\end{array}\right]$ e $l_{j}^{\prime}=\left[\begin{array}{lll}c_{j 1} & c_{j 2} & \ldots\end{array}\right.$ $\left.c_{j(m-1)} c_{i m}\right]$. Se $\tau=0$, não se troca os genes. Desta forma a nova prole é construída. Este operador de cruzamento tem a peculiaridade que é poder a partir de dois progenitores, gerarem múltiplos descendentes.

Non-Uniform Mutation: Seja $l_{n}=\left[\begin{array}{lllll}c_{n 1} & c_{n 2} & \ldots & c_{n(m-1)} & c_{n m}\end{array}\right]$ o $n$-ésimo indivíduo (cromossomo) da população de certa geração, onde $n$ é o número de indivíduos da população. Suponha

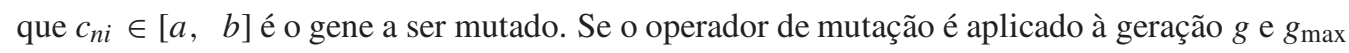
é o número máximo de gerações, então o gene gerado é dado por:

$$
c_{n i}^{\prime}= \begin{cases}c_{n i}+\Delta\left(g, b-c_{n i}\right) & \text { se } \tau=0 \\ c_{n i}-\Delta\left(g, a+c_{n i}\right) & \text { se } \tau=1\end{cases}
$$

onde,

$$
\Delta(g, y)=y\left(1-r^{\left(1-\frac{g}{g \max }\right)^{\beta}}\right),
$$

e $r$ é um número aleatório no intervalo $(0,1], \beta$ é um parâmetro a ser escolhido que determina o grau de dependência do número de gerações e $\Delta(g, y)$ retorna valores no intervalo $(0, y]$ tal 
que a probabilidade de retornar valor próximo de zero aumenta à medida que as gerações vão aumentando. A perturbação provocada no valor do gene por este operador de mutação é menor com o passar das gerações $g$. Esta propriedade faz com que este operador faça busca uniforme no espaço inicial quando $g$ é pequeno, e busca próxima dos valores dos genes quando $g \longrightarrow g_{\max }$, favorecendo o ajuste fino. A Figura 2 ilustra o fluxo de processamento do algoritmo genético de codificação real clássico (AGCR) utilizado neste trabalho.

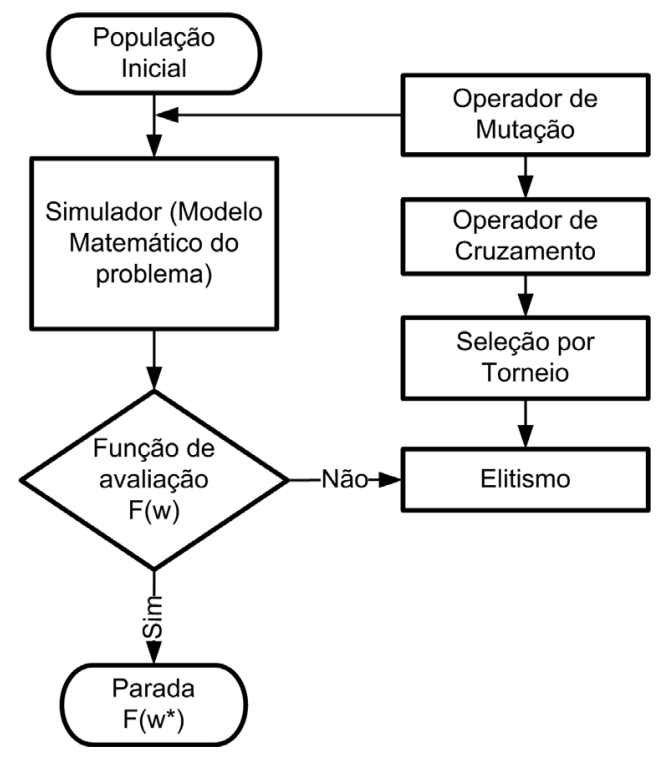

Figura 2: Fluxograma do algoritmo genético de codificação real clássico (AGCR).

\section{METODOLOGIA}

O operador proposto neste trabalho, denominado de Operador de Evolução Orientada (OEO), tem o objetivo de acelerar o processo de convergência do AGCR, na busca do indivíduo otimizado. O OEO consiste de operador genético que faz uso da função interpoladora-extrapoladora.

Considere o AGCR definido anteriormente aplicado ao processo de otimização. A função interpoladora-extrapoladora, denotada por $E$, será definida após cada determinado intervalo de gerações. Cada um desses intervalos, denota-se por:

$$
M=\left[c_{i, j}\right]_{n \times m},
$$

a matriz cuja linha $l_{i}=\left[c_{i, 1}, c_{i, 2}, \ldots, c_{i, m}\right], i=1,2, \ldots, n$, é formada pelos genes do melhor indivíduo da geração, onde este melhor indivíduo é melhor que todos os melhores indivíduos das gerações anteriores. Neste caso, a função de avaliação do AGCR, denotada por $F$, será sempre estritamente decrescente quando calculada nas linhas de $M$. Isto é, $F\left(l_{i+1}\right)<F\left(l_{i}\right)$, $i=1, \ldots, n-1$. Assim, o número $n$ de linhas de $M$, é igual ao número de gerações onde foi encontrado o melhor indivíduo, com valores estritamente decrescentes da função de avaliação. O número $m$ de colunas de $M$ representa o número de genes. 
Denota-se por $c_{j}=\left[c_{1, j}, c_{2, j}, \ldots, c_{n, j}\right]^{T}$ a $j$-ésima coluna da matriz $M$, e por $x_{i}=F\left(l_{n+1-i}\right)$, $i=1,2, \ldots, n$, os valores em ordem crescente da função de avaliação calculados nas linhas de $M$. Assim, tem-se que,

$$
F\left(l_{n}\right)=x_{1}<x_{2}<\cdots<x_{m}=F\left(l_{1}\right) .
$$

Para cada coluna $c_{j}$ de genes da matriz $M$ é definida uma função interpoladora-extrapoladora $E_{j}$ do seguinte modo: o domínio de $E_{j}$ deve ser o intervalo fechado $[q, r]$, contendo todos os valores da função de avaliação calculados nas linhas de $M$, isto é, contendo os valores $x_{1}, \ldots, x_{m}$. $\mathrm{O}$ extremo superior $r$ do intervalo, sem perda de generalidade, pode ser igual a $x_{m}=F\left(l_{1}\right)$. $\mathrm{O}$ extremo inferior $q$ deve ser definido aleatoriamente, de tal modo que,

$$
q<F\left(l_{n}\right) \cdot \delta
$$

onde $1-\delta$ é o percentual de progresso desejado, o qual deve ser menor que o menor valor da função de avaliação no intervalo de gerações considerado. A imagem de $E_{j}$ deve conter todos os genes da coluna $c_{j}$, de tal modo que,

$$
E_{j}\left(x_{i}\right)=y_{i j},
$$

onde $y_{i j}=c_{(n+1-i) j}, i=1,2, \ldots, n$. Finalmente, a função $E_{j}$ é definida através de interpolação suave entre os pontos $\left(x_{i}, y_{i j}\right)$ e $\left(x_{i+1} y_{(i+1) j}\right), i=1,2, \ldots, n-1$ e extrapolação para o subintervalo $\left[q, x_{1}\right]$ do domínio. O valor $E_{j}(q)$ é o gene melhorado na posição $j$ correspondente ao intervalo de gerações considerado, Figura 3.

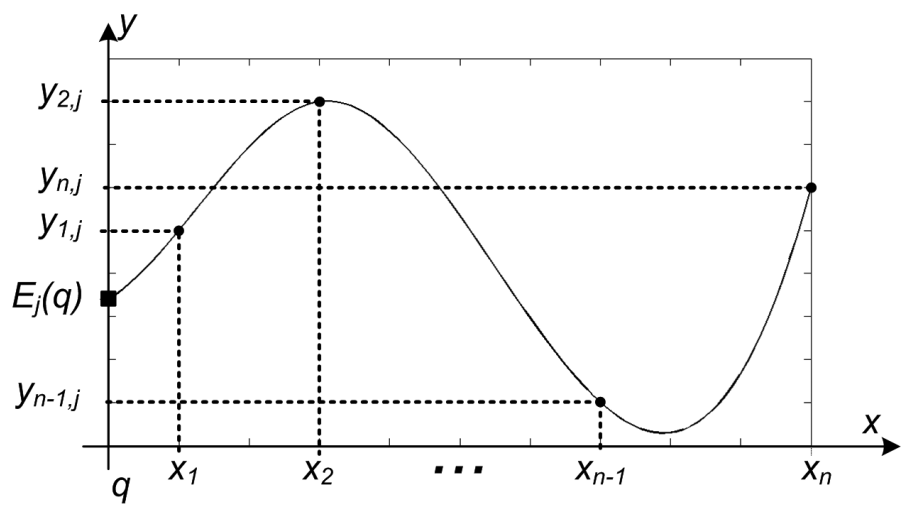

Figura 3: $E_{j}(q)$, gene extrapolado na posição $j$ correspondente ao intervalo de gerações considerado, quadrado sobre $x=0$.

Vários métodos de extrapolação podem ser considerados, como por exemplo, Piecewise Cubic Interpolation, Cubic Spline Interpolation.

Em (3.2) aparece uma imposição onde, da geração $g$ para a geração $g+i$, se não for gerado novo indivíduo melhor, caso que ocorre com frequência nos AG, não se deve guardar em $M$ o mesmo indivíduo da geração $g$ anterior, pois, isto ocasionaria problema numérico ao proceder 
a extrapolação. O operador proposto (OEO) permite construir novo conjunto de dados a partir do conjunto discreto de dados previamente conhecidos nos cromossomos de cada indivíduo, garantindo continuidade e carregando os padrões intrínsecos de cada gene para o único gene.

\section{VALIDAÇÃO EM GEOPROSPECÇÃO}

A validação do operador OEO será realizada em problemas de geoprospecção, que consiste na obtenção dos parâmetros do processo de estratificação do solo em $N$-camadas [11]. Os parâmetros a serem obtidos são: o número de camadas $N$, a resistividade elétrica $\rho_{i}$ e a espessura $h_{i}$ de cada camada $i$.

Na aplicação, utiliza-se a metodologia desenvolvida por F.A. Wenner [10], onde quatro eletrodos igualmente espaçados de distância $a$ sobre uma reta são cravados no solo e a corrente elétrica $I$ é injetada em um dos eletrodos externo e colhida no outro, como ilustrado na Figura 4, sendo medida a diferença de potencial $V$ entre os dois eletrodos internos [12].

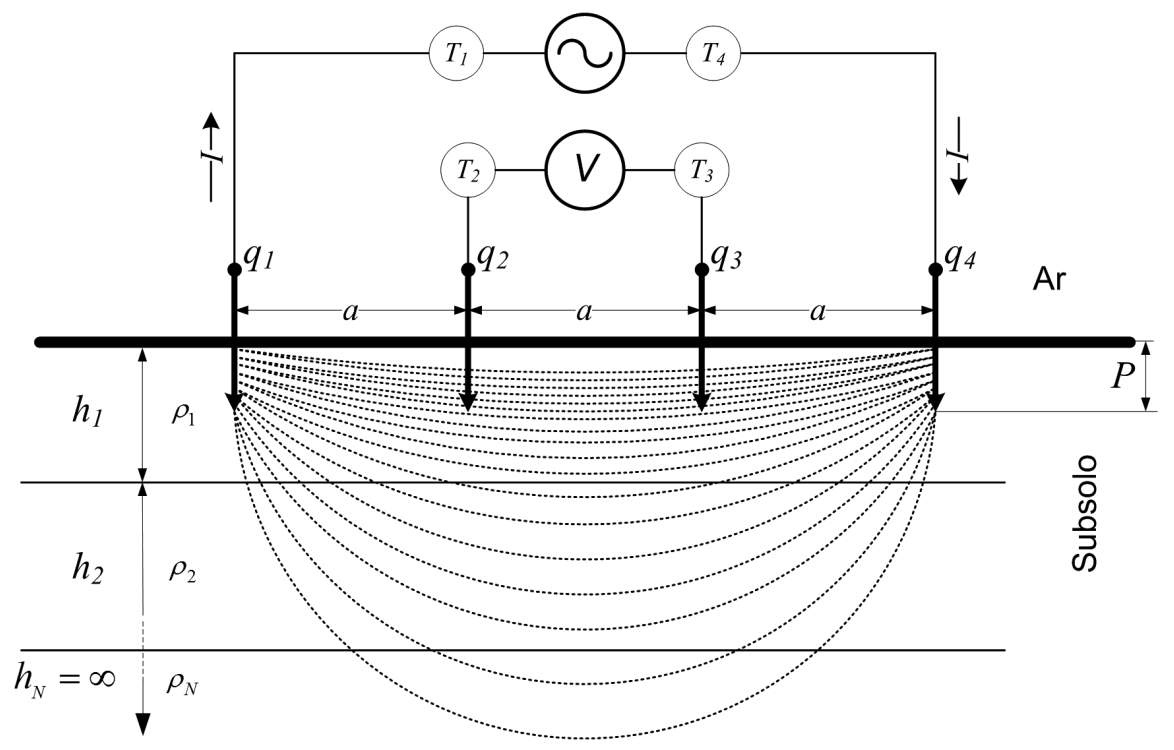

Figura 4: Método de Wenner para coleta de dados em prospecção geoelétrica.

Utilizando o valor da corrente $I$ (que é conhecido) e da diferença de potencial $V$ medida, é calculada a resistência aparente $R_{m}$. Através deste método, variando a distância $a$, é possível produzir a curva de resistividade aparente experimental $\rho_{a E}(a)$, dada pela expressão:

$$
\rho_{a E}(a)=\frac{4 \pi a \cdot R_{m}}{1+\frac{2 a}{\sqrt{a^{2}+4 P^{2}}}-\frac{2 a}{\sqrt{4 a^{2}+4 P^{2}}}}
$$

em (4.1) $P$ é a profundidade dos eletrodos. De posse dos dados coletados é possível produzir a estratificação do solo. Vários métodos que aproximam os resultados são utilizados. Matematicamente, este é um problema inverso pois, não pode ser resolvido analiticamente sem uso de aproximações. 
E.D. Sunde [13] desenvolve forma analítica para através dos dados encontrados da estratificação, ou seja, a curva de resistividade aparente experimental $\rho_{a E}(a)$, construir uma curva de resistividade aparente teórica $\rho_{a T}(a)$. O algoritmo de Sunde utiliza o equacionamento de propagação de ondas em meios estratificados, transformando o problema inverso em um problema direto. A Figura 5 ilustra o processo de estratificação do solo utilizado neste trabalho.

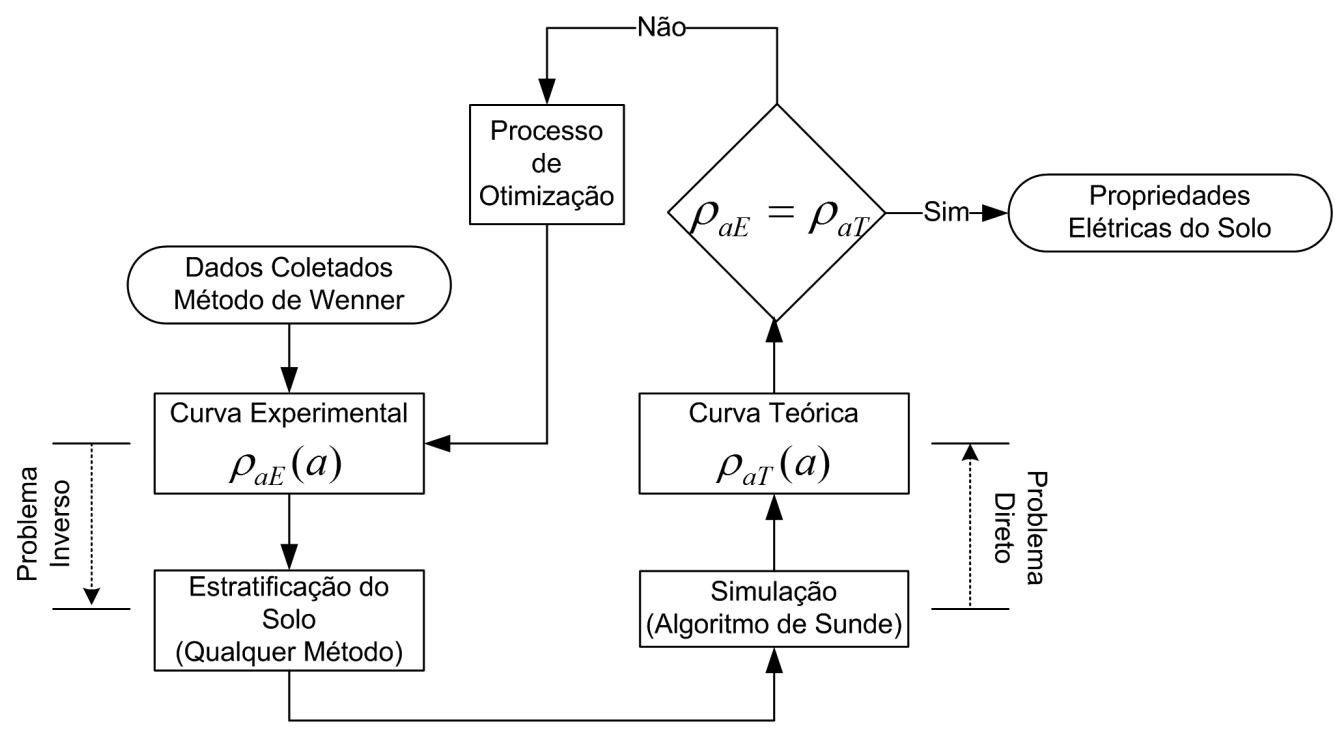

Figura 5: Fluxo de dados no processo de estratificação do solo.

Portanto, através de métodos aproximados de estratificação do solo pode-se produzir curvas de resistividades aparentes experimentais e, partindo do algoritmo de Sunde e dos parâmetros obtidos por método de otimização, é possível produzir a curva de resistividade aparente teórica $\rho_{a T}(a)$, dada pela expressão:

$$
\rho_{a T}(a)=2 \rho_{1} \cdot a \cdot \int_{0}^{\infty} N_{N}(m) \cdot\left[J_{0}(m a)-J_{0}(2 m a)\right] d m
$$

onde $N_{N}$ é a função característica que define a estrutura heterogênea do solo e $J_{0}$ é a função de Bessel de primeira espécie ordem zero, $m$ é a variável auxiliar de integração. Assim, a cada iteração, uma curva de resistividade aparente teórica $\rho_{a T}(a)$ é comparada com a curva de resistividade aparente experimental $\rho_{a E}(a)$ através da função de avaliação dada pela métrica:

$$
F(w)=\sum_{i=1}^{k}\left\|\frac{\rho_{a E\left(a_{i}\right)}-\rho_{a T\left(a_{i}\right)}}{\rho_{a E\left(a_{i}\right)}}\right\|, \rho_{a E\left(a_{i}\right)}, \rho_{a T\left(a_{i}\right)} \in \mathrm{R}, w \in \mathrm{R}^{2 \cdot N-1},
$$

onde $k$ é a quantidade de elementos do vetor $a$, ou seja, o número de pontos sobre a curva $\rho_{a E}(a)[11]$. 


\section{RESULTADOS}

Utilizando o AGCR apresentado na Seção 2, inclui em seus operadores o OEO. Este AGCR que contém o OEO nas suas sub-rotinas será chamado de algoritmo genético com operador de evolução (AGOE). A cada geração $g+i$, definida a priori, o OEO é executado.

Foram realizados quatro estudos de casos como aplicação para validação. Estes estudos foram retirados de trabalho conceituados e publicados, que utilizaram outros métodos de otimização no processo de geoprospecção. O número $N$ de camadas foi fixo conforme os resultados contidos em cada trabalho. Em alguns destes trabalhos estavam dispostas as curvas de resistividades aparentes teóricas $\rho_{a T}(a)$. Nos trabalhos onde as curvas de resistividades aparentes teóricas $\rho_{a T}(a)$ não estavam dispostas, foi utilizado o algoritmo de Sunde (que obtém $\rho_{a T}(a)$ através dos resultados) para obter esta curva, assim todos os resultados utilizam a mesma função de avaliação $F(w)$. Todos os resultados obtidos pelo AGOE na solução dos problemas práticos, tiveram os seguintes parâmetros definidos: tamanho do torneio $\tau_{t}=3$, taxa de mutação inicial $T_{m i}=5 \%$, taxa de mutação final $T_{m f}=40 \%$, taxa de cruzamento inicial $T_{c i}=80 \%$, taxa de cruzamento final $T_{c f}=20 \%$, número máximo de geração $g_{\max }=100$, número de indivíduo na população $n=20$ e a execução do OEO de 10 em 10 gerações.

\subsection{Estudo de Caso I}

Este estudo foi retirado do trabalho de Gonos \& Stathopulos [17] onde os autores utilizam o Classical Binary Genetic Algorithm (CBGA). Os autores realizam vários testes com resultados encontrados em outros trabalhos e tem a metodologia desenvolvida com base em pacote de software. Normalmente é definido a priori o número de camadas do solo a ser estratificado. Porém, este procedimento não é o correto, uma vez que o número de camadas $N$ é um dos parâmetros a ser otimizado. Neste trabalho Gonos \& Stathopulos consideraram a estratificação com 2-camadas.

Com a mesma curva de resistividade aparente experimental do trabalho [17], Dawalibi \& Blattner [12] resolvem o mesmo problema de otimização, no entanto, utilizando técnica de interpretação que inclui curvas gráficas em conjunto com software que utiliza o Method of SteepestDescent (MSD) ao qual a curva de resistividade aparente experimental $\rho_{a E}(a)$ pode ser diretamente comparada com várias curvas padrões. Estes autores consideram também esta estratificação com 2-camadas.

Na Tabela 1 são apresentados os valores dos espaçamentos entre os eletrodos $a$, os valores da resistividade aparente experimental $\rho_{a E}(a)$ e os valores das resistividades aparente teóricas $\rho_{a T}(a)$ encontradas pelos três métodos, AGOE, CBGA e o MSD. São apresentados na Tabela 2 os desvios entre os valores da resistividade aparente experimental $\rho_{a E}(a)$ e os valores das resistividades aparente teóricas $\rho_{a T}(a)$ para cada método, estes desvios são calculados utilizando a expressão (4.3).

Na Figura 6 são ilustradas as curvas de resistividades aparentes experimental e teóricas do Caso I. 
Tabela 1: Valores das resistividades aparentes experimental e teóricas para os métodos AGOE, CBGA \& MSD.

\begin{tabular}{|c|c|c|c|c|}
\hline $\begin{array}{c}a \\
{[m]}\end{array}$ & $\begin{array}{c}\rho_{a E} \\
{[\Omega \cdot m]}\end{array}$ & $\begin{array}{c}\rho_{a T} \text { AGOE } \\
{[\Omega \cdot m]}\end{array}$ & $\begin{array}{c}\rho_{a T} \text { CBGA } \\
{[\Omega \cdot m]}\end{array}$ & $\begin{array}{c}\rho_{a T} \text { MSD } \\
{[\Omega \cdot m]}\end{array}$ \\
\hline 2.5 & 327.43 & 327.40 & 319.99 & 326.94 \\
5.0 & 233.88 & 233.79 & 233.12 & 233.60 \\
7.5 & 187.43 & 187.41 & 186.47 & 187.35 \\
10.0 & 168.03 & 168.05 & 165.94 & 168.03 \\
12.5 & 159.53 & 159.52 & 156.62 & 159.52 \\
15.0 & 155.35 & 155.33 & 151.99 & 155.34 \\
\hline
\end{tabular}

Tabela 2: Valores dos desvios para os métodos AGOE, CBGA \& MSD.

\begin{tabular}{|c|c|c|c|}
\hline $\begin{array}{c}a \\
{[m]}\end{array}$ & $\begin{array}{c}\text { Desvio [\%] } \\
\text { AGOE }\end{array}$ & $\begin{array}{c}\text { Desvio [\%] } \\
\text { CBGA }\end{array}$ & $\begin{array}{c}\text { Desvio [\%] } \\
\text { MSD }\end{array}$ \\
\hline 2.5 & 0.0070 & 2.2722 & 0.1497 \\
5.0 & 0.0374 & 0.3250 & 0.1197 \\
7.5 & 0.0097 & 0.5122 & 0.0427 \\
10.0 & -0.0127 & 1.2438 & 0.0000 \\
12.5 & 0.0039 & 1.8241 & 0.0063 \\
15.0 & 0.0070 & 2.1629 & 0.0064 \\
\hline$F(w)$ & 0.0776 & 8.3402 & 0.3248 \\
\hline
\end{tabular}

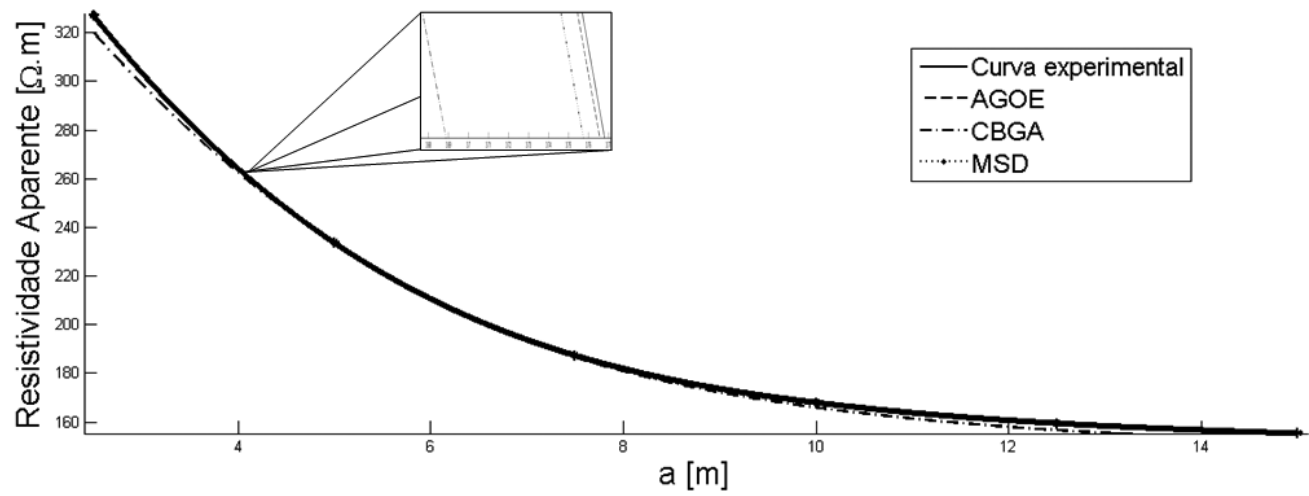

Figura 6: Curvas de resistividades aparentes Estudo de Caso I.

Considerando o desvio total para estratificação do solo entorno dos $20 \%$, ou seja, $F(w)=20$ como sendo razoável [11], observa-se na Tabela 2, que o desvio total obtido pelo CBGA entorno de $8 \%$ é satisfatório. Este mesmo caso foi resolvido utilizando o MSD e o desvio total obtido ficou entorno de $0.3 \%$. Contudo, o resultado obtido pelo AGOE ficou ainda menor, entorno de $0.07 \%$ (veja Tab. 1 e Tab. 2). Observa-se na Figura 6, que a curva de resistividade aparente experimental tem o comportamento não sinuoso, o que facilita o processo de otimização. $\mathrm{Na}$ Tabela 3 estão dispostos os resultados da estratificação dos três métodos utilizados. 
Tabela 3: Resultados das estratificações do solo - Caso I.

\begin{tabular}{|c|c|c|c|}
\hline $\begin{array}{c}\text { Parâmetros } \\
\text { Otimizados }\end{array}$ & $\begin{array}{c}\text { AGOE } \\
\text { Proposto }\end{array}$ & $\begin{array}{c}\text { CBGA } \\
{[12]}\end{array}$ & $\begin{array}{c}\text { MSD } \\
{[17]}\end{array}$ \\
\hline$\rho_{1}[\Omega \cdot m]$ & 383.63 & 367.73 & 383.00 \\
$\rho_{2}[\Omega \cdot m]$ & 147.69 & 143.56 & 147.70 \\
\hline$h_{1}[m]$ & 2.56 & 2.70 & 2.56 \\
$h_{2}[m]$ & $\infty$ & $\infty$ & $\infty$ \\
\hline
\end{tabular}

\subsection{Estudo de Caso II}

A segunda aplicação foi retirada do trabalho de del Alamo [15]. Neste trabalho o autor utiliza como métodos de otimização o Gradient Technique with Method Newton-Raphson (GTNR). Em seu trabalho, del Alamo compara seu resultado com resultados de outros métodos e considera seus resultados de estratificação para este solo aceitáveis, estratificando em 2-camadas.

Com a mesma curva de resistividade aparente experimental utilizada por de del Alamo [15], Lagacé et al. [16] realiza novos estudos utilizando o Least-Squared Minimization Techniques (LSMT). No entanto, Lagacé et al. consideram esta estratificação com 3-camadas enquanto del Alamo [15] considera a mesma estratificação com 2-camadas.

Na Tabela 4 são apresentados os valores dos espaçamentos entre os eletrodos $a$ e os valores da curva de resistividade aparente experimental $\rho_{a E}(a)$, ainda na Tabela 4 estão apresentados os valores das curvas de resistividades aparentes teóricas $\rho_{a T}(a)$ encontrados por cada método, tanto para 2-camadas como para 3-camadas. A Tabela 5 dispõem os valores dos desvios produzidos pelos três métodos, tanto para 2-camadas como para 3-camadas.

Tabela 4: Valores das resistividades aparentes experimental e teóricas para os métodos AGOE, GTNR \& LSMT.

\begin{tabular}{|c|c|c|c|c|c|}
\hline $\begin{array}{c}a \\
{[m]}\end{array}$ & $\begin{array}{c}\rho_{a E}(a) \\
{[\Omega \cdot m]}\end{array}$ & $\begin{array}{c}\rho_{a T} \text { AGOE } \\
\text { 2-Camadas } \\
{[\Omega \cdot m]}\end{array}$ & $\begin{array}{c}\rho_{a T} \text { GTNR } \\
{[\Omega \cdot m]}\end{array}$ & $\begin{array}{c}\rho_{a T} \text { AGOE } \\
\text { 3-Camadas } \\
{[\Omega \cdot m]}\end{array}$ & $\begin{array}{c}\rho_{a T} \text { LSMT } \\
{[\Omega \cdot m]}\end{array}$ \\
\hline 1.0 & 136.0 & 136.00 & 141.77 & 135.95 & 130.99 \\
2.0 & 140.0 & 151.79 & 168.77 & 149.95 & 147.32 \\
4.0 & 214.0 & 214.47 & 253.71 & 212.24 & 214.95 \\
10.0 & 446.0 & 407.46 & 456.81 & 438.45 & 439.85 \\
20.0 & 685.0 & 607.46 & 631.25 & 681.79 & 693.69 \\
40.0 & 800.0 & 800.00 & 769.85 & 797.10 & 809.58 \\
\hline
\end{tabular}

São ilustradas na Figura 7 as curvas de resistividades aparentes experimental e teóricas para todos os métodos de otimização utilizados no Caso II.

Observa-se nos resultados disposto na Tabela 5 que quando se faz estratificação do solo e não se considera o número de camadas $N$ como parâmetros a ser otimizado, pode-se levar a resultados 
Tabela 5: Valores dos desvios para os métodos AGOE, GTNR \& LSMT.

\begin{tabular}{|c|c|c|c|c|}
\hline $\begin{array}{c}a \\
{[m]}\end{array}$ & $\begin{array}{c}\text { Desvio [\%] } \\
\text { AGOE } \\
\text { 2-Camadas }\end{array}$ & $\begin{array}{c}\text { Desvio [\%] } \\
\text { GTNR }\end{array}$ & $\begin{array}{c}\text { Desvio [\%] } \\
\text { AGOE } \\
\text { 3-Camadas }\end{array}$ & $\begin{array}{c}\text { Desvio [\%] } \\
\text { LSMT }\end{array}$ \\
\hline 1.0 & 0.0000 & -4.2490 & 0.0360 & 3.6806 \\
2.0 & -8.4266 & -20.5549 & -7.1108 & -5.2323 \\
4.0 & -0.2204 & -18.5583 & 0.8193 & -0.4485 \\
10.0 & 8.6412 & -2.4339 & 1.6924 & 1.3777 \\
20.0 & 11.3187 & 7.8461 & 0.4677 & -1.2692 \\
40.0 & 0.0000 & 3.7683 & 0.3621 & -1.1982 \\
\hline$F(w)$ & 28.6070 & 57.4005 & 10.4883 & 13.2065 \\
\hline
\end{tabular}

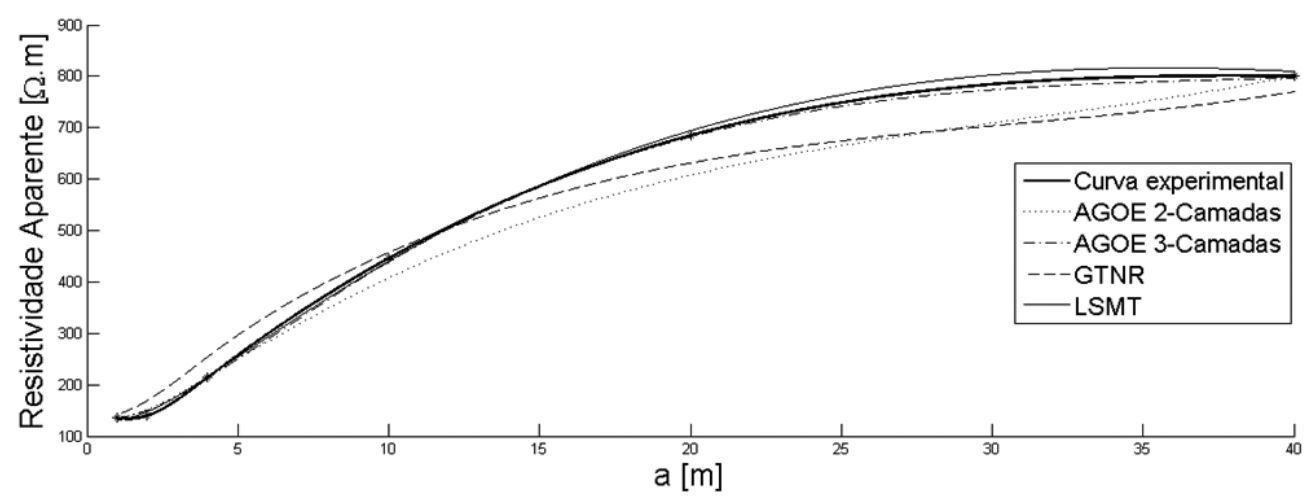

Figura 7: Curvas de resistividades aparentes Estudo de Caso II.

não aceitáveis na prática. Em del Alamo [15], foi considerado a estratificação em 2-camadas, obtendo desvio total entorno de $57 \%$ para o método GTNR, enquanto o AGOE fica entorno de $28 \%$, que ainda é uma valor elevado para o desvio. Tendo como base os mesmos dados, porém considerando a estratificação em 3-camadas, obtém-se para o LSMT [16], o desvio total entorno de $13 \%$, enquanto o AGOE resulta em um desvio total entorno dos $10 \%$, o que já é aceitável na prática.

Comparando os resultados do AGOE para a estratificação do Caso II, em 2-camadas e em 3camadas, observa-se a diferença no desvio total entorno de $63.34 \%$ menor para o caso onde é considerado a estratificação em 3-camadas, ou seja, este solo corresponde ao modelo de solo estratificado horizontalmente, se for considerado com mais de 3-camadas. A Tabela 6 dispõem os resultados da estratificação do Caso II para os três métodos.

Observe que nos resultados, Tabela 6 , as variações nos valores de $\rho_{1}$ fica entorno de $5.87 \%$ entre os diferentes métodos, considerando tanto 2-camadas como 3-camadas, enquanto as variações nos valores de $\rho_{2}$ chegam a $64.31 \%$. Isto ocorre porque as funções a serem otimizadas correspondem a modelos não lineares e a quantidade de parâmetros define a curva a ser otimizada. 
Tabela 6: Resultados das estratificações do solo - Caso II.

\begin{tabular}{|c|c|c|c|c|}
\hline $\begin{array}{c}\text { Parâmetros } \\
\text { Otimizados }\end{array}$ & $\begin{array}{c}\text { AGOE } \\
\text { 2-Camadas }\end{array}$ & $\begin{array}{c}\text { GTNR } \\
{[15]}\end{array}$ & $\begin{array}{c}\text { AGOE } \\
\text { 3-Camadas }\end{array}$ & $\begin{array}{c}\text { LSMT } \\
{[16]}\end{array}$ \\
\hline$\rho_{1}[\Omega \cdot m]$ & 133.07 & 135.98 & 133.50 & 128.00 \\
$\rho_{2}[\Omega \cdot m]$ & 1033.04 & 890.42 & 2495.00 & 1930.00 \\
$\rho_{3}[\Omega \cdot m]$ & - & - & 257.10 & 520.00 \\
\hline$h_{1}[m]$ & 3.00 & 2.32 & 3.40 & 3.10 \\
$h_{2}[m]$ & $\infty$ & $\infty$ & 15.00 & 15.00 \\
$h_{3}[m]$ & - & - & $\infty$ & $\infty$ \\
\hline
\end{tabular}

\subsection{Estudo de Caso III}

Esta aplicação foi retirada do trabalho de del Alamo [14]. Neste trabalho o autor aplica várias técnicas de otimização em alguns problemas de estratificação. Para o caso aqui apresentado, os resultados obtidos em [14] utilizou a First Order Gradient Technique (FOGT), este trabalho considera a estratificação do solo em 2-camadas.

São apresentados na Tabela 7 os valores dos espaçamentos entre os eletrodos $a$, os valores das resistividades aparente experimental $\rho_{a E}(a)$ e os valores encontrados para as resistividades aparentes teóricas produzidos pelo AGOE e o FOGT. Na Tabela 8 estão dispostos os valores encontrados para os desvios entre as curvas experimental e teóricas utilizando os dois métodos.

Tabela 7: Valores das resistividades aparentes experimental e teóricas para os métodos AGOE \& FOGT.

\begin{tabular}{|c|c|c|c|}
\hline $\begin{array}{c}a \\
{[m]}\end{array}$ & $\begin{array}{c}\rho_{a E} \\
{[\Omega \cdot m]}\end{array}$ & $\begin{array}{c}\rho_{a T} \text { AGOE } \\
{[\Omega \cdot m]}\end{array}$ & $\begin{array}{c}\rho_{a T} \text { FOGT } \\
{[\Omega \cdot m]}\end{array}$ \\
\hline 2.5 & 320.0 & 320.00 & 297.31 \\
5.0 & 245.0 & 226.62 & 234.34 \\
7.5 & 182.0 & 182.00 & 191.84 \\
10.0 & 162.0 & 163.79 & 169.79 \\
12.5 & 168.0 & 155.86 & 158.61 \\
15.0 & 152.0 & 152.00 & 152.67 \\
\hline
\end{tabular}

Neste estudo, o desempenho do AGOE leva à solução com valor da função de avaliação 42.84\% menor que o valor da função de avaliação do FOGT. Na Tabela 9 encontram-se os resultados obtidos para esta estratificação. Na Figura 8 são ilustradas as curvas de resistividades aparentes experimental e teóricas do Caso III.

Observa-se na Tabela 9 que a discrepância entre os valores dos parâmetros (resultados da estratificação) encontrados, é de $12.68 \%$ em média, enquanto a diferença da função de avaliação entre os métodos fica entorno de $42.84 \%$. Isto é indicativo de que pequena variação nos valores dos parâmetros provoca grande variação entre as curvas $\rho_{a E}(a)$ e $\rho_{a T}(a)$. 
Tabela 8: Valores dos desvios para os métodos AGOE \& FOGT.

\begin{tabular}{|c|c|c|}
\hline$a$ & Desvio [\%] & Desvio [\%] \\
{$[\mathrm{m}]$} & AGOE & FOGT \\
\hline 2.5 & 0.0000 & 7.0892 \\
5.0 & 7.4988 & 4.3494 \\
7.5 & 0.0000 & -5.4082 \\
10.0 & -1.1059 & -4.8101 \\
12.5 & 7.2205 & 5.5875 \\
15.0 & 0.0000 & -0.4414 \\
\hline$F(w)$ & 15.8253 & 27.6858 \\
\hline
\end{tabular}

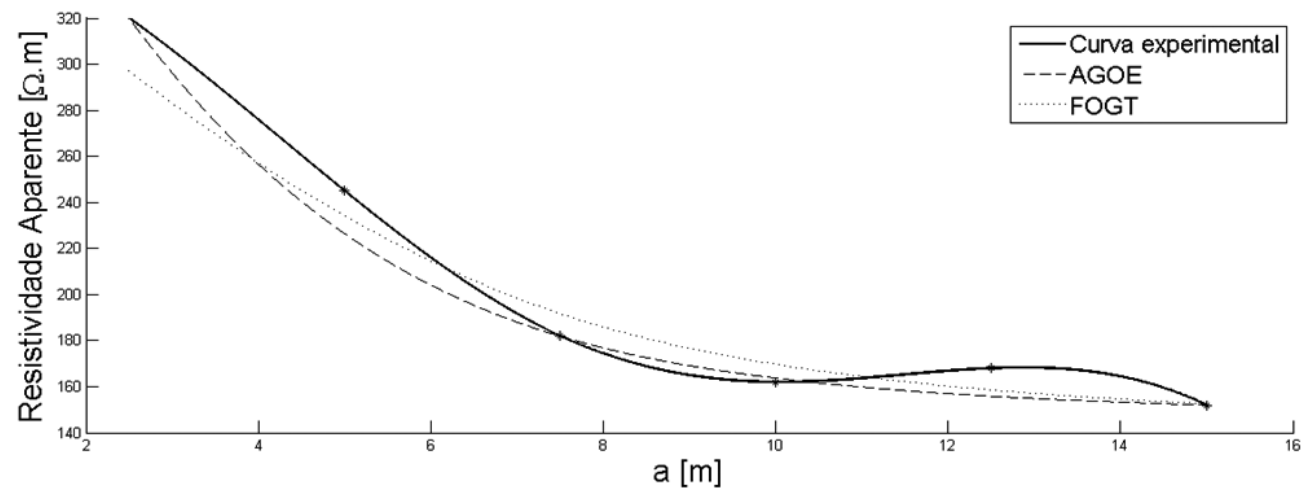

Figura 8: Curvas de resistividades aparentes Estudo de Caso III.

Tabela 9: Resultados das estratificações do solo - Caso III.

\begin{tabular}{|c|c|c|}
\hline $\begin{array}{c}\text { Parâmetros } \\
\text { Otimizados }\end{array}$ & $\begin{array}{c}\text { AGOE } \\
\text { Proposto }\end{array}$ & $\begin{array}{c}\text { FOGT } \\
{[14]}\end{array}$ \\
\hline$\rho_{1}[\Omega \cdot m]$ & 378.38 & 324.60 \\
$\rho_{2}[\Omega \cdot m]$ & 144.90 & 141.45 \\
\hline$h_{1}[m]$ & 2.49 & 3.17 \\
$h_{2}[m]$ & $\infty$ & $\infty$ \\
\hline
\end{tabular}

\subsection{Estudo de Caso IV}

O quarto estudo foi retirado do trabalho de Gonos \& Stathopulos [17]. Neste trabalho os autores utilizam o Classical Binary Genetic Algorithm (CBGA) e consideram o solo estratificado em 3-camadas. Usando a mesma curva de resistividade aparente experimental $\rho_{a E}(a)$ dada em [17], resolve-se o problema de otimização utilizando o AGCR e o AGOE, os resultados são então comparados.

A Tabela 10 dispõem os valores dos espaçamentos entre os eletrodos $a$, os valores das resistividades aparente experimental $\rho_{a E}(a)$ e os valores das resistividades aparentes teóricas $\rho_{a T}(a)$ 
produzidos pelos três métodos, AGOE, CBGA e o AGCR. Na Tabela 11 estão disposto os valores calculados dos desvios.

Tabela 10: Valores das resistividades aparentes experimental e teóricas para os métodos AGOE, CBGA \& AGCR.

\begin{tabular}{|c|c|c|c|c|}
\hline $\begin{array}{c}a \\
{[m]}\end{array}$ & $\begin{array}{c}\rho_{a E} \\
{[\Omega \cdot m]}\end{array}$ & $\begin{array}{c}\rho_{a T} \text { AGOE } \\
{[\Omega \cdot m]}\end{array}$ & $\begin{array}{c}\rho_{a T} \text { CBGA } \\
{[\Omega \cdot m]}\end{array}$ & $\begin{array}{c}\rho_{a T} \text { AGCR } \\
{[\Omega \cdot m]}\end{array}$ \\
\hline 1.0 & 214.0 & 214.06 & 203.51 & 211.27 \\
3.0 & 256.0 & 255.16 & 250.93 & 242.90 \\
5.0 & 273.0 & 290.43 & 282.50 & 274.64 \\
10.0 & 307.0 & 306.86 & 294.37 & 295.96 \\
15.0 & 284.0 & 284.05 & 274.82 & 279.41 \\
20.0 & 250.0 & 257.93 & 252.74 & 256.75 \\
30.0 & 225.0 & 222.31 & 222.06 & 222.70 \\
50.0 & 210.0 & 196.08 & 198.47 & 195.08 \\
80.0 & 186.0 & 187.18 & 190.00 & 185.05 \\
\hline
\end{tabular}

Tabela 11: Valores dos desvios para os métodos AGOE, CBGA \& AGCR.

\begin{tabular}{|c|c|c|c|}
\hline $\begin{array}{c}a \\
{[m]}\end{array}$ & $\begin{array}{c}\text { Desvio [\%] } \\
\text { AGOE }\end{array}$ & $\begin{array}{c}\text { Desvio [\%] } \\
C B G A\end{array}$ & $\begin{array}{c}\text { Desvio [\%] } \\
\text { AGCR }\end{array}$ \\
\hline 1.0 & -0.0287 & 4.8899 & 1.2750 \\
\hline 3.0 & 0.3243 & 1.9775 & 5.1170 \\
\hline 5.0 & -6.3854 & -3.4820 & -0.6017 \\
\hline 10.0 & 0.0437 & 4.1133 & 3.5932 \\
\hline 15.0 & -0.0191 & 3.2299 & 1.6143 \\
\hline 20.0 & -3.1744 & -1.0985 & -2.7036 \\
\hline 30.0 & 1.1920 & 1.3043 & 1.0186 \\
\hline 50.0 & 6.6270 & 5.4892 & 7.1041 \\
\hline 80.0 & -0.6392 & -2.1544 & 0.5056 \\
\hline$F(w)$ & 18.4339 & 27.7489 & 23.5332 \\
\hline
\end{tabular}

Os parâmetros encontrados pelo AGOE produz desvio de $33.57 \%$ menor quando comparados com o valor do desvio obtido pelo CBGA. O mesmo valor do desvio total obtido pelo AGOE, agora em comparação com o AGCR, é de aproximadamente 21.67\% menor (veja Tab. 11).

Na Figura 9 são ilustradas as curvas de resistividades aparentes experimental e teóricas do estudo de Caso IV e na Tabela 12 encontra-se os resultados obtidos pelos três métodos para esta estratificação.

Nesta estratificação, foi utilizado o AGCR na tentativa de obter resultados de comparação entre tempo de execução e número de gerações utilizando o OEO. A Tabela 13 apresenta alguns valores encontrados no desempenho dos dois métodos (AGOE e AGCR). 


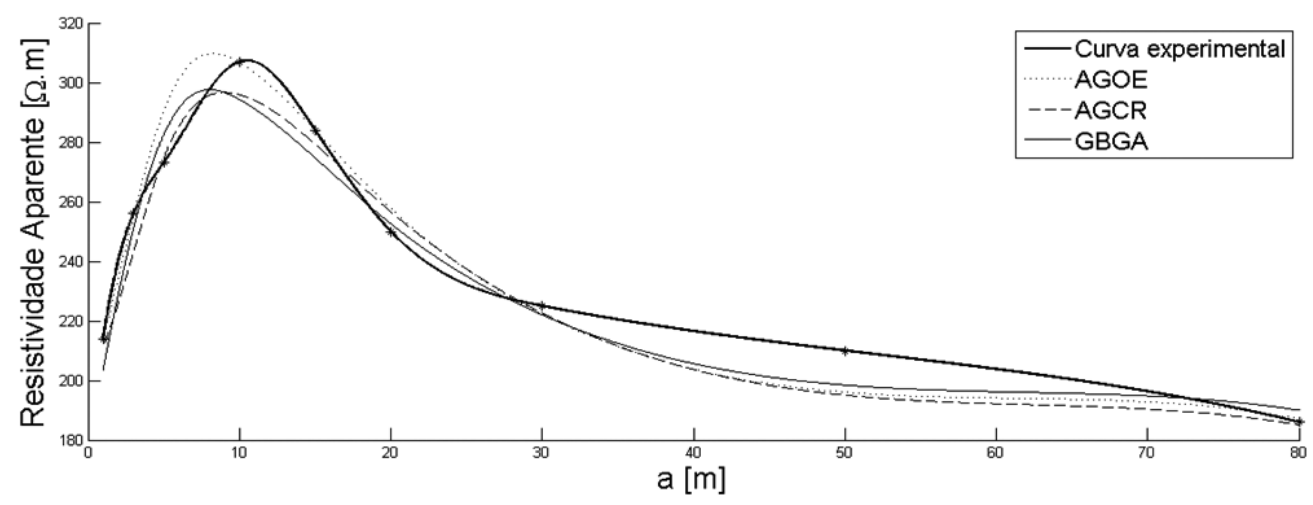

Figura 9: Curvas de resistividades aparentes Estudo de Caso IV.

Tabela 12: Resultados das estratificações do solo - Caso IV.

\begin{tabular}{|c|c|c|c|c|}
\hline $\begin{array}{c}\text { a } \\
{[m]}\end{array}$ & $\begin{array}{c}\text { Parâmetros } \\
\text { Otimizados }\end{array}$ & AGOE & $\begin{array}{c}\text { CBGA } \\
{[17]}\end{array}$ & AGCR \\
\hline 2.5 & $\rho_{1}[\Omega \cdot m]$ & 209.76 & 196.90 & 208.40 \\
5.0 & $\rho_{2}[\Omega \cdot m]$ & 395.60 & 351.70 & 375.49 \\
7.5 & $\rho_{3}[\Omega \cdot m]$ & 182.33 & 185.20 & 179.53 \\
\hline 10.0 & $h_{1}[m]$ & 2.12 & 1.70 & 2.39 \\
12.5 & $h_{2}[m]$ & 7.33 & 8.40 & 7.93 \\
15.0 & $h_{3}[m]$ & $\infty$ & $\infty$ & $\infty$ \\
\hline
\end{tabular}

Tabela 13: Avaliação entre o AGOE e o AGCR.

\begin{tabular}{|c|c|c|c|c|}
\hline Método & $n$ & $g$ & $t$ [minuto] & $F\left(w^{*}\right)$ \\
\hline AGOE & 20 & 100 & 9.08 & 18.4339 \\
AGCR & 20 & 100 & 8.02 & 23.5332 \\
\hline
\end{tabular}

Nos dados da Tabela 13, é observado que o AGCR obteve o tempo menor de execução em relação ao AGOE. Como o AGOE=AGCR + OEO, o tempo que o AGOE levou a mais para executar esta tarefa foi exatamente o tempo de processamento do OEO. Com isto, a diferença de 1.06 minutos que o AGOE gastou a mais, $11.67 \%$ do tempo total gasto pelo AGCR, foi utilizado na execução do OEO, embora o AGOE tenha reduzido o valor de $F\left(w^{*}\right)$ em $21.67 \%$.

\section{COMENTÁRIOS}

Para o valor do número $q$ em (3.3), observou-se que, quando o valor de $q$ está próximo do valor de $F\left(l_{n}\right)$, ele produz um indivíduo melhor que o melhor já existente. Ou no pior dos casos, ele irá produzir um indivíduo bem adaptado que se não for o melhor, irá doar seus genes na produção de indivíduos melhores adaptados. Quando o valor de $q$ fica distante do valor de $F\left(l_{n}\right)$ e muito 
próximo do valor de $F(w)$, os indivíduos gerados podem ter o valor da função de avaliação distante do valor desejado. Neste trabalho utilizou-se $\delta=0.95$.

Outro procedimento útil é, quando o OEO for executado desprezar os 5 primeiros indivíduos da matriz $M$ em (3.1). A ideia de desprezar os primeiros indivíduos parte do pressuposto que no início do processo de otimização, os indivíduos gerados e guardados na matriz $M$, não consegue apresentar evidências de padrão na coluna dos genes. A Figura 10 ilustra valores de $F(w)$ para alguns indivíduos gerados pelo OEO e pelo AGCR apenas nas gerações onde o OEO foi executado. Observe que no início quando o valor de $g$ é pequeno, o valor de $F(w)$ encontrados pelo OEO estão distantes do valor de $F(w)$ encontrado pelo AGCR, no entanto, com o passar das gerações, o padrão vai ficando evidente e os indivíduos encontrados pelo OEO tem o valor de $F(w)$ menor.

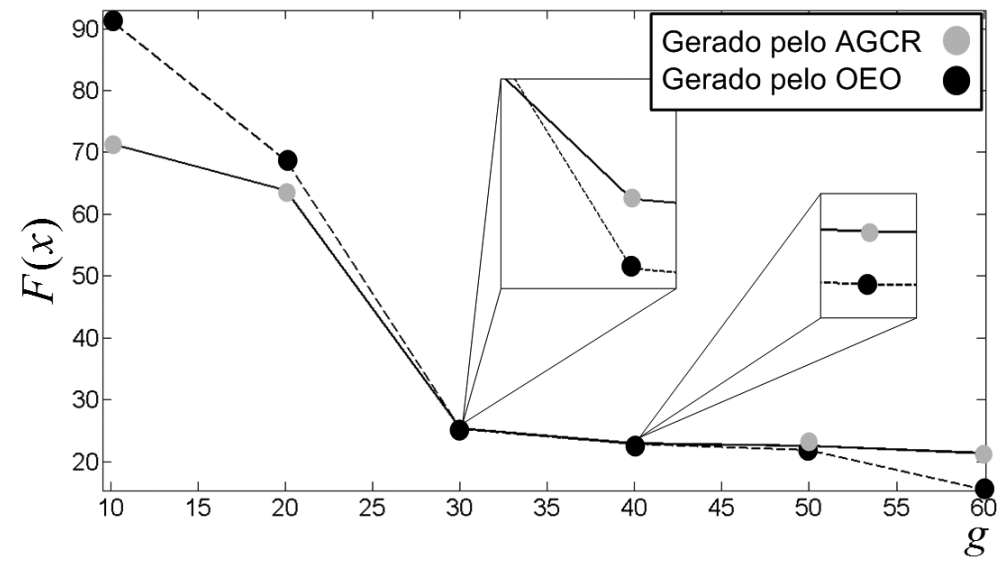

Figura 10: Comparação entre soluções geradas (indivíduos).

Observando ainda a Figura 10, neste caso em especial, a partir da $30^{\mathrm{a}}$ geração, ou seja, a partir da terceira execução do OEO, 50\% dos melhores indivíduos nas gerações múltiplas de 10 foram encontradas pelo OEO. Outro fator observado foi, com a utilização do OEO o fenômeno de estagnação foi diminuído nos casos simulados pois quando ele não encontra indivíduo melhor que o da geração corrente, ele produz genes com boas características que podem, através do operador de cruzamento, fazer parte de novo indivíduo com alto fitness.

Analisando a Tabela 13 e sabendo que o OEO foi utilizado de 10 em 10 gerações, tomando $g_{\max }=100$, tem-se que em média, para o processo de estratificação o OEO leva 6.36 segundos em cada execução enquanto o tempo médio gasto em cada geração é de 5.44 segundos.

Observa-se que o AGOE levou tempo maior que o AGCR na solução do problema. No entanto, para todos os casos práticos simulados, o critério de parada utilizado foi $g=100$. Para estes casos, os valores de $F(w)$ obtidos pelo AGOE são menores. Deve-se também, levar em conta que, quanto mais parâmetros a serem otimizados tiver o problema, mais tempo será gasto na execução do OEO. 


\title{
7 CONCLUSÃO
}

A característica de reconhecer a direção de desenvolvimento do gene pelo OEO, analisada através da matriz $M$, acelerou o processo de convergência do método de otimização. O AGOE em relação ao AGCR, reduziu o número de gerações necessárias para encontrar o valor do $F\left(w^{*}\right)$.

Como em processo de estratificação horizontal do solo é difícil encontrar terreno que corresponda a $F\left(w^{*}\right)=10^{-3}$, todos os problemas aqui propostos pararam utilizando o critério número de gerações. No entanto, o AGOE obteve valores menores em relação aos outros métodos testados (CBGA, MSD, GTNR, LSMT, FOGT e AGCR) com redução dos desvio, encontrando sempre melhor curva de resistividade teórica.

O OEO promoveu estímulo em direção ao ótimo no AGOE, reduzindo o valor da função de avaliação encontrada e conseguiu reunir as características intrínsecas de cada gene na produção de novo gene, gerando novo indivíduo melhor adaptado.

\section{AGRADECIMENTOS}

Os autores agradecem a Coordenação de Aperfeiçoamento de Pessoal de Nível Superior (Capes), Ministério da Educação (MEC), pelo suporte financeiro através da bolsa de estudo Proc. no. BEX 3873/10-2. O terceiro autor, Jesus Carlos da Mota, agradece a FAPEG - Fundação de Amparo à Pesquisa do Estado de Goiás pelo apoio financeiro, projeto Universal 05/2012.

\begin{abstract}
This work presents a genetic operator developed from a mathematical curve extrapolation method. This operator help the production of fitter individual within a real coded genetic algorithm in which inherent patters of the best individuals of each generations are recognized. The operator is compared to five alternative optimization techniques applied to geoelectrical prospection.
\end{abstract}

Keywords: optimization, mathematical operator, genetic algorithm, real coding.

\section{REFERÊNCIAS}

[1] G.E.P. Box \& J.S. Hunter. Multifactor experimental designs for exploring response surfaces. Annals of Mathematical Statistics, 28(1) (1957), 195-241.

[2] J.H. Holland. Adaptation in Natural and Artificial Systems. The University of Michigan Press, Ann Arbor, MI (1975).

[3] Z. Michalewicz. Genetic Algorithms + Data Structures = Evolution Programs. Springer, Charlotte, North Carolina, USA (1992).

[4] F. Herrera \& M. Lozano. Adaptive genetic operators based on coevolution with fuzzy behaviors. IEEE Transactions on Evolutionary Computation, 5(2) (2001), 149-165.

[5] W.P. Calixto, J.C. da Mota \& B.P. Alvarenga. Methodology for the Reduction of Parameters in the Inverse Transformation of Schwarz-Christoffel Applied to Electromagnetic Devices with Axial 
Geometry. International Journal of Numerical Modelling: Electronic Networks, Devices and Fields, doi: 10.1002/jnm.804, (2011).

[6] L.M. de Campos; J.A. Gamez \& S. Moral. Partial abductive inference in Bayesian belief networks an evolutionary computation approach by using problem-specific genetic operators. IEEE Transactions on Evolutionary Computation, 6(2) (2002), 105-131.

[7] S.Y. Yuen \& C.K. Chow. A Genetic Algorithm That Adaptively Mutates and Never Revisits. IEEE Transactions on Evolutionary Computation, 13(2) (2009), 454-472.

[8] W.P. Calixto, E.G. Marra, L.C. Brito \& B.P. Alvarenga. A New Methodology to Calculate Carter Factor Using Genetic Algorithms. International Journal of Numerical Modelling: Electronic Networks, Devices and Fields, 24(4) (2010), 387-399.

[9] F. Herrera, M. Lozano \& J.L. Verdegay. Tackling Real-Coded Genetic Algorithms: Operators and Tools for Behavioural Analysis. Artificial Intelligence Review, 12 (1998), 265-319.

[10] F.A. Wenner. Method of Measuring Earth Resistivity. Bulletin of the National Bureau of Standards, (1916).

[11] W.P. Calixto, L. Martins Neto, M. Wu \& K. Yamanaka. Parameters Estimation of a Horizontal Multilayer Soil Using Genetic Algorithm. IEEE Transactions on Power Delivery, 25(3) (2010), 1250-1257.

[12] F. Dawalibi \& C.J. Blattner. Earth Resistivity Measurement Interpretation Techniques. IEEE Transactions on Power Apparatus and Systems, PAS-103(2) (1984), 374-382.

[13] E.D. Sunde. Earth conduction effects in transmission systems. MacMilan, New York (1968).

[14] J.L. del Alamo. A Comparison among Eight Different Techniques to Achieve an Optimum Estimation of Electrical Grounding Parameters in Two-Layered Earth. IEEE Transactions on Power Delivery, 8(4) (1993), 1890-1899.

[15] J.L. del Alamo. A Second Order Gradient Technique for an Improved Estimation of Soil Parameters in a Two-Layer Earth. IEEE Transactions on Power Delivery, 6(3) (1991), 1166-1170.

[16] P.J. Lagacé, J. Fortin \& E.D. Crainic. Interpretation of Resistivity Sounding Measurements in N-Layer Soil using Electrostatic Images. IEEE Transactions on Power Delivery, 11(3) (1996), 1349-1354.

[17] I.F. Gonos \& I.A. Stathopulos. Estimation of Multilayer Soil Parameters Using Genetic Algorithms. IEEE Transactions on Power Delivery, 20(1) (2005), 100-106. 